\title{
Genetical Studies of Non-flagellate Mutants of Salmonella
}

\author{
By T. IINO AND M. ENOMOTO \\ National Institute of Genetics, Misima, Japan
}

(Received 8 July 1965)

\begin{abstract}
SUMMARY
Transductions were carried out with 22 non-flagellate mutants originating spontaneously from Salmonella typhimurium LT 2 . Taking the productions of swarm and trail as the criteria of recombination and complementation respectively, the mutants were classified into seven groups, namely $f l a A, f l a B, f l a C, f l a D, f l a F, f l a J$ and $f a K$. Each of them may correspond to a cistron. Among them, $f a A, B, C, D$ and $J$ were co-transduced with the structural gene of phase-1 flagellin, H1. Based on the frequency of recombination between two non-flagellate mutants, and that of $\mathrm{H1}$ co-transduction when available, linearly arranged maps of mutant sites in $f a A, f l a C$ and $f l a F$ were constructed. Co-linearity was demonstrated between the recombination map and complementation map in $f l a A$ and $f l a C$, but with some exceptions in flaF. Partial complementation was frequently observed in combination of two mutants in a cistron.

Transductions from thirteen non-flagellate mutants investigated by Joys \& Stocker to the above mutants indicated that, among the five complementation groups assigned by them on their mutants, four correspond to our flaA, flaB, flaC and flaD; the remaining one, flaE, is missing in our mutants. Similarly, among eleven mutant sites of the non-flagellate strains of Salmonella abortusequi sL23, ten are distributed in $\mathrm{flaA}$, flaF and $\mathrm{flaC}$. The remaining one, assigned to a mutant of $\mathrm{flaG}$, does not belong to any $f l a$ cistrons described above. The recovery of flagellation in the $f a G$ strain by transduction was unsuccessful even when a flagellate strain was used as a donor.

Rabbits were immunized with each of the twenty-one stable non-flagellate mutant clones, and their ability to elicit antiserum specific to flagellar protein was examined by absorption-agglutination test. Among the clones examined, only one belonging to $f a G$ was found to elicit antiserum specific in flagellar antigen of the parental flagellate strain. It was inferred that the flaG mutant can synthesize flagellin but cannot construct flagellar fibres. The remaining fla mutants were presumed to be unable to synthesize flagellin.
\end{abstract}

\section{INTRODUCTION}

Among the genes which control flagellar formation in the genus Salmonella, there is a group of genes called $f l a$ (flagellation gene). The characteristic of $f a$ is that a mutation of a $f l a$ gene from $f a^{+}$to $f a^{-}$results in the loss of the ability to produce flagella in both flagellar phase-1 and -2. Detailed investigation of them promises to disclose a refined genetic regulatory system for bacterial flagellation.

Genetic studies of $f l a$ genes have been undertaken immediately after the discovery of transduction in salmonella-P 22 system (Stocker, Zinder \& Lederberg, 1953). The studies have been extended in parallel at Dr B. A. D. Stocker's laboratory in the

Vol. 43, No. 2, was issued 31 May 1966 
Lister Institute of Preventive Medicine, London, England, and at our laboratory with mutual exchange of information.

The present paper deals with the complementation and linkage analyses of salmonella $f l a$ genes. It may be read as the counterpart of the paper by Joys \& Stocker (1965).

\section{METHODS}

Non-flagellate mutants used for the present experiment originated spontaneously from the following two motile flagellate strains: Salmonella typhimurium LT2 $i: 1,2$ and $S$. abortusequi sL $23(a): e, n, x . \chi$-phage and/or semisolid medium were used for the selection of these mutants. With the exception of some specific serotypes, $\chi$-phage infects motile salmonellas; both non-flagellate and paralysed organisms are resistant to this bacteriophage (Meynell, 1961). During the present investigation, an efficient selective method was invented by using $\chi$-phage and semisolid medium, so as to distinguish non-flagellate mutant clones from other non-motile mutants, namely paralysed, immediately on selective medium. The method was described elsewhere (Enomoto \& Iino, 1963a). In addition, a nonflagellate strain of $S$. paratyphi $B$, sw 543 (b:-) (Stocker et al. 1953) was used. Together with the origin and characteristics of these non-flagellate mutants, the presence or absence of revertants is listed in Table 1 . In order to compare the result of complementation tests done by Joys \& Stocker (1965) with ours, eleven non-flagellate mutants of $S$. typhimurium investigated by them were also used.

The general procedure of cultivation, observation of motility, identification of antigen type and transduction of motility character were according to the methods of Stocker et al. (1953) and Lederberg \& Iino (1956).

Salmonella phage P22 grown on indicated host cultures was used as transducing phage. The method to screen flagellate motile transductional clones was modified as follows. A lysate of phage propagated from donor bacteria and the broth culture of a recipient were mixed in a test tube, and left for $15 \mathrm{~min}$. at $24^{\circ}$. The mixture was then diluted tenfold into broth and brushed in plates of semisolid medium by means of a $1 \mathrm{ml}$. sterilized glass syringe. The adequate volume for one brush was $0.05 \mathrm{ml}$. The inoculated plates were kept at $37^{\circ}$ for $6-8 \mathrm{hr}$, then at $23^{\circ}$ for $12 \mathrm{hr}$, and numbers of swarms and trails developed were scored under binocular microscope at $\times 15$ magnification. A swarm resulting from the transduction is regarded to correspond to a flagellate recombinant clone. Trail production in transduction of motility character is a typical example of unilinear inheritance (Lederberg, 1956; Stocker, 1956). It represents the transmission of a non-replicated genetic fragment through many generations to a single descendant as a heterogenote. Consequently, the production of trail may be used as a criterion of complementation between two flagellation genes; that is, in cases where trails are produced from a transduction between two non-flagellate mutants, mutant sites in these two strains are presumed to be in different functional units.

For the immunization of rabbit with salmonella organisms, bacterial cultures in broth at the late logarithmic phase were washed and resuspended in formol saline to a final concentration of $10^{6}$ bacteria per ml. The suspensions were kept overnight at room temperature and thereafter injected intraperitoneally the first time and intravenously from the second time. A total of $6 \mathrm{ml}$. of bacterial sus- 
pension was given to each rabbit in six injections. Then the rabbit was bled and the serum was processed. The titre of antiserum was determined by tube agglutination test following the procedure of Kauffman (1954).

\section{RESULTS}

Grouping of non-flagellate mutants by complementation test

Transduction from a motile strain of Salmonella typhimurium, LT2, to each of the 22 non-flagellate mutants originated from it produced both trails and swarms on semisolid plates. The ratio of the number of trails to that of swarms was 6 to 10 .

Transductions of $f a$ genes were next made in all pairwise combinations of these non-flagellate mutants. Numbers of trails and swarms were scored on each combination, and graded into four as regards the degree of either trail or swarm

Table 1. Non-flagellate mutants of Salmonella used for the experiments

\begin{tabular}{|c|c|c|c|c|c|}
\hline Mutant no. & $\begin{array}{l}\text { Comple- } \\
\text { menta- } \\
\text { tion } \\
\text { group }\end{array}$ & $\begin{array}{l}\text { Rever- } \\
\text { sion }\end{array}$ & $\begin{array}{c}\text { Other } \\
\text { characters }\end{array}$ & Strain no. & $\mathrm{fla}^{+}$parent \\
\hline *fla-21 & C & + & ath pro & SL 480 & \\
\hline * fla-22 & $\mathbf{A}$ & - & ath pro & SL481 & \\
\hline fla-23 & $\mathbf{A}$ & + & ath pro & SL 482 & \\
\hline${ }^{*} f l a-24$ & F & + & ath pro & SL 483 & \\
\hline *fla-25 & $\mathbf{F}$ & - & cys & sL 488 & \\
\hline$f l a-26$ & $\mathbf{F}$ & - & ade pro & SL 490 & \\
\hline *fla-28 & $\mathbf{J}$ & + & . & sw1154 & \\
\hline$* f l a-31$ & $\mathbf{C}$ & + & . & sJ 31 & \\
\hline *fla-32 & $\mathbf{F}$ & + & . & sJ 33 & \\
\hline *fla-33 & $\mathbf{F}$ & + & . & SJ 34 & \\
\hline$* f l a-34$ & $\mathbf{A}$ & + & . & SJ 35 & S. typhimurium \\
\hline fla-35 & $\mathrm{F}$ & + & . & sJ 36 & LT2 $i: 1 \cdot 2$ \\
\hline$* f a-36$ & $\mathbf{B}$ & + & . & sJ79 & \\
\hline$f a-37$ & $\mathbf{A}$ & + & . & SJ 80 & \\
\hline fla-38 & $\mathbf{C}$ & + & . & SJ 81 & \\
\hline fla-39 & $\mathbf{A}$ & - & . & sJ 82 & \\
\hline$f l a-40$ & $\mathbf{F}$ & + & . & SJ 84 & \\
\hline$f l a-41$ & $\mathbf{A}$ & - & . & SJ 373 & \\
\hline fla-42 & $\mathbf{D}$ & - & . & SJ 374 & \\
\hline$f a-43$ & F & + & . & SJ 376 & \\
\hline$f l a-44$ & $\mathbf{K}$ & - & . & SJ 400 & \\
\hline$f l a-45$ & $\mathbf{F}$ & + & . & SJ 408 & \\
\hline$f l a-69$ & $\mathbf{A}$ & - & gal & sw543 & S. paratyphi $B$ b:- \\
\hline *fla-100 & $\mathbf{G}$ & - & • & SJ 28 & \\
\hline fla-101 & $\mathbf{F}$ & + & . & SJ 148 & \\
\hline *fla-102 & $\mathbf{F}$ & - & . & SJ 149 & \\
\hline *fa-103 & $\mathbf{F}$ & + & . & SJ 150 & \\
\hline *fla-104 & $\mathbf{F}$ & + & . & SJ 152 & S. abortusequi \\
\hline *fla-105 & C & + & . & SJ 153 & SL $23(a): e n x$ \\
\hline *fla-106 & $\mathbf{F}$ & - & . & sJ 154 & \\
\hline *fla-107 & $\mathbf{F}$ & - & . & SJ 156 & \\
\hline *fla-108 & $\mathbf{F}$ & - & . & SJ 157 & \\
\hline *fla-109 & $\mathbf{A}$ & + & . & SJ 158 & \\
\hline *fa-110 & $\mathbf{A}$ & + & . & SJ 160 & \\
\hline
\end{tabular}

*Mutants used for the test of flagellin CRM production.

Symbols : $a t h=$ adenine + thiamine requirement; ade = adenine requirement; pro = proline requirement; $c y s$ = cystine requirement; gal = galactose not fermentated. 
formation. Grade-0 produces no transductional clones at all while in the control transduction experiment, in which the original flagellate strain LT 2 was used as the donor, about 100 swarms and more than 500 trails were scored. In Grade-1, transductional clones were produced less than $10 \%$ of the control. Grade-2 represents the production of transductional clone between 10 and $50 \%$ of the control, and Grade-3 50-100\%. The results of grading are shown in Table 2. Except fla-25, the grades obtained in reciprocal transductions were identical or differed at most one grade in every combination. The evidence that $f l a-25$ is a presumable deletion mutant will be given later.

\section{Table 2. Grouping of non-flagellate mutants of Salmonella typhimurium $L T 2$ by transduction tests}

Numbers outside and inside of parentheses show degree of swarm and trail production respectively. grade-0: no transductional clone appeared; grade-1: transductional clones were produced less than $10 \%$ of the control; grade-2: between 10 and $50 \%$; grade-3: between 50 to $100 \%$. All combinations not listed here give grade-3.

\begin{tabular}{|c|c|c|c|c|c|c|c|c|c|c|c|c|}
\hline \multirow[b]{2}{*}{ Recipient } & \multicolumn{7}{|c|}{$\begin{array}{c}\text { Group A } \\
\text { Donor }\end{array}$} & \multicolumn{5}{|c|}{$\begin{array}{c}\text { Group C } \\
\text { Donor }\end{array}$} \\
\hline & $A-37$ & $A-23$ & $A-34$ & $A-39$ & $A-22$ & $A-41$ & LT 2 & Recipient & $C-31$ & $C-38$ & $C-21$ & LT 2 \\
\hline$A-37$ & $\cdot$ & $2(2)$ & $2(3)$ & $2(3)$ & $2(3)$ & $1(1)$ & $3(3)$ & $C-31$ & • & $2(1)$ & $3(3)$ & $\mathbf{3}(\mathbf{3})$ \\
\hline$A-23$ & $2(2)$ & . & $1(0)$ & $1(0)$ & $2(2)$ & $1(0)$ & $3(3)$ & $C-38$ & $2(2)$ & . & $2(0)$ & $3(3)$ \\
\hline$A-34$ & $2(2)$ & $1(0)$ & . & $1(0)$ & $2(2)$ & $1(0)$ & $3(3)$ & $C-21$ & $3(3)$ & $2(0)$ & . & 3 (3) \\
\hline A-39 & $2(3)$ & $1(0)$ & $1(0)$ & 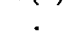 & $1(0)$ & $0(0)$ & $3(3)$ & $\operatorname{mot} A-206$ & $3(3)$ & 3 (3) & $3(3)$ & 3 (3) \\
\hline$A-22$ & $2(3)$ & $2(2)$ & $2(2)$ & $1(0)$ & 18 & $0(0)$ & $3(3)$ & & & & & \\
\hline$A-41$ & $0(0)$ & $0(0)$ & $0(0)$ & $0(0)$ & $0(0)$ & . & $3(3)$ & & & & & \\
\hline $\operatorname{mot} A-206$ & $3(3)$ & $3(3)$ & 3 (3) & $3(3)$ & $3(3)$ & $\mathbf{3}(3)$ & $3(3)$ & & & & & \\
\hline
\end{tabular}

\begin{tabular}{|c|c|c|c|c|c|c|c|c|c|c|}
\hline \multirow[b]{2}{*}{ Recipient } & \multicolumn{10}{|c|}{$\begin{array}{c}\text { Group F } \\
\text { Donor }\end{array}$} \\
\hline & $F-33$ & $F-32$ & $F-45$ & F-26 & $F-35$ & $F-40$ & $F-43$ & F-24 & F- 25 & LT 2 \\
\hline $\boldsymbol{F}-33$ & ${ }^{\circ}$ & $2(1)$ & $3(2)$ & $3(2)$ & $3(2)$ & $3(3)$ & 3 (3) & $3(3)$ & $2(1)$ & $3(3)$ \\
\hline F-32 & $2(0)$ & . & $2(2)$ & $2(2)$ & $3(2)$ & $3(3)$ & $3(3)$ & $3(3)$ & $2(0)$ & $3(3)$ \\
\hline$F-45$ & $1(1)$ & $0(1)$ & . & $1(2)$ & $2(2)$ & $2(3)$ & $2(3)$ & $2(3)$ & $0(0)$ & $3(3)$ \\
\hline$F-26$ & $2(1)$ & $1(0)$ & $1(0)$ & . & $2(0)$ & $2(1)$ & $2(2)$ & $2(2)$ & $0(0)$ & $3(3)$ \\
\hline$F-35$ & $2(1)$ & $2(1)$ & $2(1)$ & $1(1)$ & . & $2(2)$ & $2(2)$ & $2(2)$ & $2(0)$ & $3(\mathbf{3})$ \\
\hline$F-40$ & $2(3)$ & $2(3)$ & $2(2)$ & $2(1)$ & $2(2)$ & . & $2(3)$ & $2(3)$ & $2(0)$ & $3(3)$ \\
\hline$F-43$ & $3(3)$ & $2(2)$ & $2(2)$ & $2(1)$ & $2(1)$ & $2(2)$ & . & $2(2)$ & $2(0)$ & $3(3)$ \\
\hline$F-24$ & $3(3)$ & $2(2)$ & $2(2)$ & $2(1)$ & $2(2)$ & $1(2)$ & $0(3)$ & & $2(0)$ & $3(3)$ \\
\hline $\boldsymbol{F}-25$ & $0(0)$ & $0(0)$ & $0(0)$ & $0(0)$ & $2(1)$ & $2(0)$ & $2(1)$ & $2(1)$ & 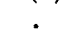 & $3(3)$ \\
\hline $\operatorname{mot} A-206$ & $3(3)$ & $3(3)$ & $3(3)$ & $3(3)$ & 3 (3) & $3(3)$ & 3 (3) & $3(3)$ & $3(3)$ & $3(3)$ \\
\hline
\end{tabular}

From these results, it is possible to classify the non-flagellate mutants into the following seven groups, such that every cross involving a pair of fla- mutants of different groups gave $50-100 \%$ of the number of swarms and/or of trails that were obtained in corresponding control crosses with $\mathrm{fla}^{+}$donors, but that every $\mathrm{fla}^{-}$ mutant of a given group gave a smaller yield of swarms and/or of trails in crosses with some at least of the other $\mathrm{fla}^{-}$mutants of that group. Group A comprised six mutants, group $\mathrm{C}$ three mutants and group $\mathrm{F}$ nine mutants; each of the four remaining groups, $\mathbf{B}, \mathbf{D}, \mathbf{J}$ and $\mathbf{K}$, comprised a single mutant each as listed in Table 2. (In a preceding review article, Iino, 1964, flaB and $f a C$ in the present 
grouping were designated as $\mathrm{flaC}$ and $\mathrm{flaB}$ respectively. These old symbols are exchanged in the present paper so as to get exact correspondence of the symbol between Joys \& Stocker (1965) and us and to avoid confusion). The groups defined by consideration of the number of swarms, i.e. rate of production of complete $\mathrm{fla}^{+}$ transductants, were identical with those defined by consideration of the number of trails, i.e. of abortive $\mathrm{fla}^{+}$transductants. In partial complementation, that is in grade-1 or -2 in trail production, the decrease of the number of trail was often accompanied by the decrease in the number of minute colonies in each trail resulting in the production of shorter ones. The absence of swarm production in combination of $f l a A-41$ with $f l a-39,-22$ and $f l a F-25$ with $f l a-45,-26$ indicates that $f l a A-41$ and flaF-25 are deletion type. Except $f l a-45$, all the mutants concerned here had zero reversion rate, so that a yield of $\mathrm{fla}^{+}$recombinants of $10^{-3}$ of that in the control cross would have been detected. Partial complementation was observed even in

\section{Table 3. Frequency of production of swarms and trails in transductions between flaA mutants of Salmonella typhimurium $L T 2$}

The numbers of swarms and trails from the mixture of $5 \times 10^{7}$ phage particles and $1 \times 10^{7}$ bacteria are scored and converted to percentages to the numbers in transduction from $\mathrm{fla}^{+}$. Percentages of trails are presented in parentheses.

\begin{tabular}{|c|c|c|c|c|c|c|c|c|}
\hline \multirow{3}{*}{$\begin{array}{c}\text { Recipient } \\
\qquad \begin{array}{c}A-37\end{array}\end{array}$} & \multicolumn{6}{|c|}{ Donor } & \multirow{2}{*}{\multicolumn{2}{|c|}{$\underbrace{\operatorname{LT} 2\left(f l a^{+}\right)}_{\% \text { Total no. }}$}} \\
\hline & \multirow[t]{2}{*}{$A-37$} & \multirow{2}{*}{$\begin{array}{c}A-23 \\
11 \cdot 4 \\
(22 \cdot 0)\end{array}$} & \multirow{2}{*}{$\begin{array}{c}A-34 \\
12 \cdot 6 \\
(64 \cdot 0)\end{array}$} & \multirow{2}{*}{$\begin{array}{c}A-39 \\
16 \cdot 9 \\
(100)\end{array}$} & \multirow{2}{*}{$\begin{array}{r}A-22 \\
\mathbf{2 3 \cdot 5} \\
(100)\end{array}$} & \multirow{2}{*}{$\begin{array}{c}A \cdot 41 \\
0 \cdot 36 \\
(0 \cdot 20)\end{array}$} & & \\
\hline & & & & & & & $\begin{array}{c}100 \\
(100)\end{array}$ & $\begin{array}{c}827 \\
(3053)\end{array}$ \\
\hline$A-23$ & $\begin{array}{c}13 \cdot 3 \\
(24 \cdot 2)\end{array}$ & & $\begin{array}{c}0 \cdot 34 \\
(0 \cdot 0)\end{array}$ & $\begin{array}{r}0.42 \\
(0.0)\end{array}$ & $\begin{array}{c}13 \cdot 3 \\
(35 \cdot 6)\end{array}$ & $\begin{array}{c}0 \cdot 078 \\
(0.002)\end{array}$ & $\begin{array}{c}100 \\
(100)\end{array}$ & $\begin{array}{c}1918 \\
(6357)\end{array}$ \\
\hline$A-34$ & $\begin{array}{c}14 \cdot 7 \\
(44 \cdot 2)\end{array}$ & $\begin{array}{c}0 \cdot 38 \\
(0 \cdot 005)\end{array}$ & & $\begin{array}{c}0.51 \\
(0.018)\end{array}$ & $\begin{array}{c}12 \cdot 5 \\
(41 \cdot 4)\end{array}$ & $\begin{array}{r}0 \cdot 17 \\
(0 \cdot 0)\end{array}$ & $\begin{array}{c}100 \\
(100)\end{array}$ & $\begin{array}{c}1406 \\
(5644)\end{array}$ \\
\hline$A-39$ & $\begin{array}{c}16 \cdot 2 \\
(80 \cdot 5)\end{array}$ & $\begin{array}{c}0.51 \\
(0 \cdot 003)\end{array}$ & $\begin{array}{c}0 \cdot 16 \\
(0 \cdot 0)\end{array}$ & & $\begin{array}{c}8 \cdot 8 \\
(0 \cdot 0)\end{array}$ & $\begin{array}{c}0 \cdot 0 \\
(0 \cdot 0)\end{array}$ & $\begin{array}{c}100 \\
(100)\end{array}$ & $\begin{array}{c}1031 \\
(3695)\end{array}$ \\
\hline$A-22$ & $\begin{array}{c}18 \cdot 7 \\
(99 \cdot 5)\end{array}$ & $\begin{array}{c}10 \cdot 5 \\
(25 \cdot 2)\end{array}$ & $\begin{array}{c}10 \cdot 0 \\
(19 \cdot 8)\end{array}$ & $\begin{array}{c}4 \cdot 6 \\
(0 \cdot 1)\end{array}$ & & $\begin{array}{c}0 \cdot 0 \\
(0 \cdot 0)\end{array}$ & $\begin{array}{c}100 \\
(100)\end{array}$ & $\begin{array}{c}1403 \\
(2933)\end{array}$ \\
\hline$A-41$ & $\begin{array}{c}0 \cdot 0 \\
(0 \cdot 0)\end{array}$ & $\begin{array}{c}0.0 \\
(0.0)\end{array}$ & $\begin{array}{c}0 \cdot 0 \\
(0 \cdot 0)\end{array}$ & $\begin{array}{c}0 \cdot 0 \\
(0 \cdot 0)\end{array}$ & $\begin{array}{c}0 \cdot 0 \\
(0 \cdot 0)\end{array}$ & & $\begin{array}{c}100 \\
(100)\end{array}$ & $\begin{array}{r}942 \\
(3972)\end{array}$ \\
\hline
\end{tabular}

the combination in which a partner is one of these deletion type. Through the frequency of flagellate recombinant, i.e. swarm production, the linear order of each mutant site in the $f a A, f l a C$ and $f l a F$ are inferred as arranged in Table 2. In $f a A$ and $f a C$, it was observed that pairs of mutants which gave high recombination rates (grade-2 or $\mathbf{- 3}$ ) also gave high yields of trails, whereas pairs giving a few swarms (grade-0 or -1), presumably because of close linkage of their $\mathrm{fla}^{-}$sites, gave few trails (grade-0 or -1). If the reduction in number of trails, as compared with the number in control crosses using a wild-type donor, was taken as a measure of the closeness of the $\mathrm{fla}^{-}$sites of two mutants, then a linear sequence of sites could be inferred from the data on frequency of trail formation, and in general this sequence was the same ('co-linear') with the order inferred from recombination frequency. In $f a F$, such co-linearity is held with exceptional combination.

In order to examine in more detail the co-linearity between the recombination 
map and the complementation map in $f a A$, transductions were repeated quantitatively between all pairwise combinations among the mutants of $f a A$. The results are summarized in Table 3 and Fig. 1. From the recombination values, the sequence of the mutant sites is inferred to be $f a-37-23-34-39-22$. Fla-23, -34 and -39 are more closely linked with each other than the other two. Fla-41 covers both fla-39 and -22. One apparent discrepancy in the frequency compared to this sequence is that when $f a-41$ is a donor, the frequency is larger in combination with fla-34 than

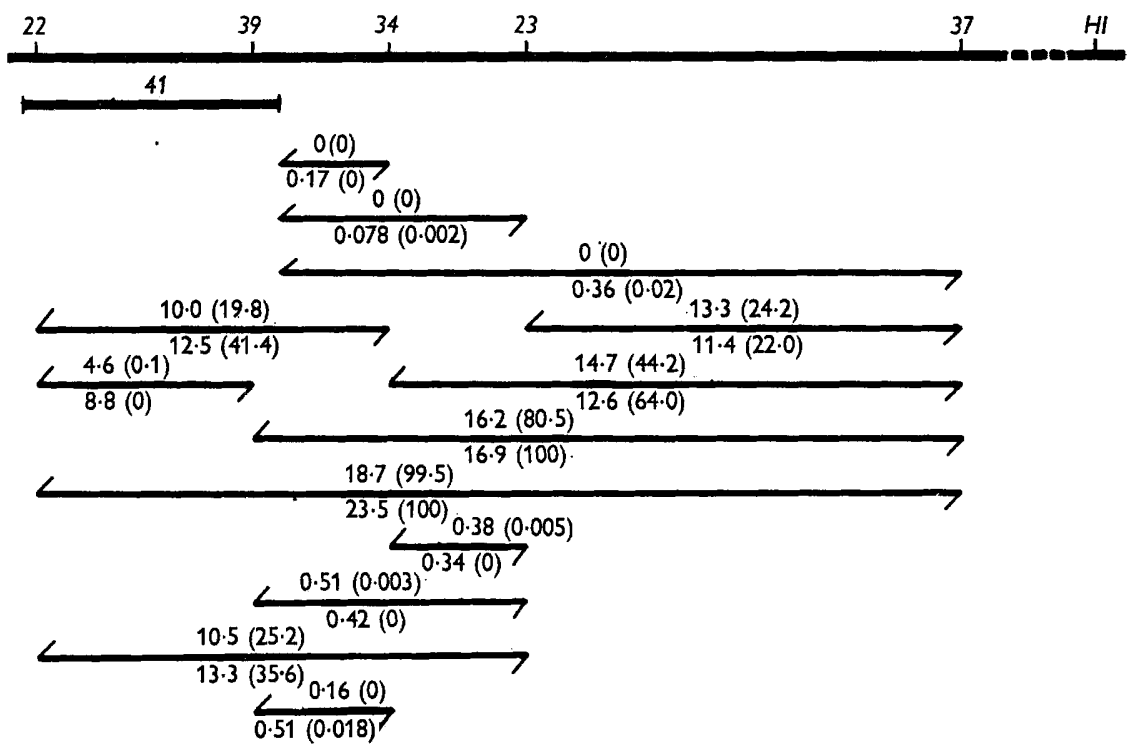

Fig. 1. Genetic map of flaA cistron in Salmonella typhimurium Ir2. Numbers above the arrow indicate recombination and complementation (in parentheses) frequencies obtained in transduction from right (donor) to left (recipient); numbers below indicate those from left to right (cf. Table 3 ).

with $f$ la-23. Statistical analysis, however, showed that this possibility could be abandoned $(t=0.32, \alpha=0.375)$. The sequential arrangement inferred from the degree of complementation agrees exactly with the above results. It is remarkable that partial complementation was observed in most of the combinations. The mutants in a cistron were not divided into subunits based on the all-or-none complementation reaction.

\section{Test of linkage between fla and $\mathrm{H} 1$ or $\mathrm{H} 2$}

Transduction of fla gene was next done with a phase-2 culture of Salmonella abony $\operatorname{cDC} 103 b: e, n, x$ as a donor and phase-1 culture of $f a^{-}$mutants as recipients. The mixture of P22 phage lysate of donor and the suspension of recipient was brushed on plates of semisolid medium containing anti- $i$ and $-1 \cdot 2$ sera. On the selection medium only those recombinants which received $H 1^{b}$ or $H 2^{e n x}$ together with $\mathrm{fla}^{+}$are expected to form swarms but those only $\mathrm{fla}^{+}$recombinants cannot. For a qualitative test of $\mathrm{fla}-\mathrm{H}$ linkage, the presence or absence of swarm production from the mixture of $3 \times 10^{8}$ phage and $10^{8}$ bacteria was examined. On semisolid 
plates, 500-2000 swarms are expected to develop from the equivalent number of recipient bacteria. Therefore, co-transduction as low as $0.05-0.2 \%$ is expected to be detected by this screening procedure. Antigen from swarm bacteria was typed by slide-agglutination test: $b$-type of swarm means that the $H 1^{b}$ was co-transduced with $f l a$ and $e n x$-type the co-transduction of $H 2^{e n x}$ with $f l a$.

As the results, it was found that mutants of $f a A, f l a B, f l a C, f l a D$ and $f l a J$ were co-transduced with $H 1$; while those of $f l a F$ and $f l a K$ were not. Co-transduction with $\mathrm{H} 2$ was not demonstrated on any $\mathrm{fla}^{-}$mutants examined.

\section{Table 4. Frequency of linked transduction between fla and $H 1$}

\begin{tabular}{|c|c|c|c|c|c|}
\hline \multirow[b]{3}{*}{$f l a^{-}$mutants } & \multirow{2}{*}{\multicolumn{3}{|c|}{ No. of recombinants tested }} & \multirow{3}{*}{$\begin{array}{c}\text { Frequency } \\
\text { of linked } \\
\text { type } \\
(\%)\end{array}$} & \multirow{3}{*}{$\begin{array}{c}99 \% \\
\text { confidence } \\
\text { interval } \\
( \pm \%)\end{array}$} \\
\hline & & & & & \\
\hline & $\begin{array}{l}\text { Linked } \\
\text { type }\end{array}$ & $\begin{array}{l}\text { Unlinked } \\
\text { type }\end{array}$ & Total & & \\
\hline$A-37$ & 48 & 321 & 369 & $13 \cdot 0$ & $4 \cdot 6$ \\
\hline$A-23$ & 41 & 340 & 381 & $10 \cdot 8$ & $4 \cdot 8$ \\
\hline$A-34$ & 33 & 265 & 298 & $11 \cdot 1$ & $5 \cdot 1$ \\
\hline$A-39$ & 48 & 385 & 433 & $11 \cdot 1$ & $4 \cdot 2$ \\
\hline$A-22$ & 16 & 378 & 394 & $4 \cdot 1$ & $2 \cdot 8$ \\
\hline$A-41$ & 221 & 115 & $\mathbf{3 3 6}$ & $66 \cdot 1$ & $7 \cdot 0$ \\
\hline$C-31$ & 13 & 238 & 251 & $5 \cdot 2$ & $4 \cdot 0$ \\
\hline$C-38$ & 2 & 296 & 298 & $0 \cdot 67$ & $1 \cdot 8$ \\
\hline$C-21$ & $\mathbf{3}$ & 363 & 366 & $0 \cdot 82$ & $1 \cdot 7$ \\
\hline$D-42$ & 4 & 233 & 237 & $1 \cdot 7$ & $2 \cdot 6$ \\
\hline$B-36$ & 1 & 280 & 281 & 0.36 & $1 \cdot 4$ \\
\hline$J-28$ & o & 184 & 184 & $<0.6$ & $1 \cdot 2$ \\
\hline
\end{tabular}

On the $H$ 1-linked $f a^{-}$mutants, transductions were repeated on semisolid plates without addition of antiserum. The developing swarms were isolated to nutrient agar plates and their antigens were typed by slide agglutination, and the frequency of co-transduction was calculated dividing the number of phase-1 donor type clones by the total number. The results are shown in Table 4. In flaA, four mutant sites, $f l a-37,-23,-34$ and -39 were transduced at the frequency of $10-13 \%$. The frequency in $f l a-41$, a deletion type, was higher than the value expected from the deletion region of the strain. The lowest frequency in $f a-22$ may indicate that fla-22 is most distal from $\boldsymbol{H} 1$ among the mutant sites examined in $f a A$. The close value in $f a-37,-23,-34$ and -39 indicates that these four mutant sites are very closely linked with each other between $H 1$ and $f l a-22$. These results agree well with those inferred from the transductions between non-flagellate mutants in the foregoing chapter.

In $f a C, f l a-31$ is more proximal to $H 1$ than $f a-38$ and -21 . Relative sequence of $f a-38$ and $f a-21$ is not clear from the present experiment. However, the most plausible sequence inferred from the data of foregoing and present chapter is H 1 fla-31 -38 -21.

As the mutant which give low recombination frequency in transduction with the mutants of two different cistrons had been detected, the relative position of $f l a A, f l a C, f l a D, f l a B$, and $f l a J$ is not known from the present experiment. If they 
are in the same side of $H 1$, the most plausible sequence is $H 1$ flaA flaC flaD flaB flaJ. The overlapping of the frequency of $H$ 1-co-transduction between the mutants of different $f a$ group, e.g. $C$ - 21 and $D-42$, leaves the possibility that some of them are in the opposite side of $H 1$.

Table 5. Transduction of fla-H 1 fragment from flaA mutants of Salmonella typhimurium $L T 2(i: 1,2)$ to a flaA-69 mutant of $S$. paratyphi $B$ SW $543(b:-)$

The number of swarm and trail gives the value obtained per $10^{7}$ donor phage particles at the multiplicity of infection (m.o.i.) of 3 . Antigen type of

\begin{tabular}{|c|c|c|c|c|c|c|}
\hline \multirow[b]{2}{*}{ Donor } & \multicolumn{2}{|c|}{ No. of transductant } & \multicolumn{2}{|c|}{ complete transductant } & \multirow{2}{*}{$\begin{array}{c}\text { Total } \\
\text { no. } \\
\text { examined }\end{array}$} & \multirow{2}{*}{$\begin{array}{c}\text { Percentag } \\
\text { of linked } \\
\text { trans- } \\
\text { duction }\end{array}$} \\
\hline & $\begin{array}{c}\text { Swarm } \\
\text { (complete) }\end{array}$ & $\begin{array}{c}\text { Trail } \\
\text { (abortive) }\end{array}$ & $\begin{array}{c}b \\
\text { (unlinked) }\end{array}$ & $\begin{array}{c}i \\
\text { (linked) }\end{array}$ & & \\
\hline$A-37$ & 40 & 217 & 173 & 3 & 176 & $1 \cdot 7$ \\
\hline$A-23$ & 23 & 0 & 279 & 3 & 282 & $1 \cdot 1$ \\
\hline$A-34$ & 18 & 0 & 202 & 1 & 203 & 0.5 \\
\hline$A-39$ & 18 & 0 & 248 & 0 & 248 & $0 \cdot 4$ \\
\hline$A-41$ & 0 & 0 & - & - & - & - \\
\hline$A-22$ & 22 & 92 & 215 & 26 & $24 ! 1$ & $10 \cdot 7$ \\
\hline LT 2 & 93 & 494 & 1215 & 137 & 1352 & $10 \cdot 1$ \\
\hline Control & 0 & 0 & - & - & - & - \\
\hline
\end{tabular}

Salmonella paratyphi $B$ sw $543 \mathrm{fla-69}$ ( $b:-)$ is known to have a mutant $f l a$ gene which is co-transduced with $H 1$ at the frequency of $10 \%$ (Lederberg \& Iino, 1956). The data in the foregoing section suggest that fla-69 is most probably in $f l a A$. The transduction from the $\mathrm{flaA}^{-}$mutants of LT2 to sw 543 was carried out to test this inference. The results were summarized in Table 5. Fla-69 does not complement $f l a-23,-34,-39$ and -41 and complements partially $f l a-37$ and -22 . Compared with the transduction from flagellated donor strain, the frequency of $H 1$ co-transduction decreased remarkably in transduction from $\mathrm{fla-37,}-23,-34$ and -39 but not from $\mathrm{fla}-22$. Thus it was indicated that $\mathrm{fla}-37,-23,-34$ and -39 are between $H 1$ and $f l a-69$ and that $f l a-22$ is distal from $H 1$ than $f l a-69$. The absence of any swarms when $f l a-69$ was treated with lysates of $\mathrm{LT} 2 \mathrm{flaA}-41$ indicates that the deletion mutation of the latter covers the site $f a-69$. Frequency of the production of $\mathrm{fla}^{+}$recombinants also fits to the above localization of $f a-69$. Summing up the results described in the foregoing and the present section, the genetic map of flaA are constructed as shown in Fig. 1.

\section{Non-flagellate mutants of Salmonella typhimurium studied by Joys \& Stocker, and originated from Salmonella abortusequi SL23}

Among the $\boldsymbol{H} 1$-linked non-flagellate mutants studied by Joys \& Stocker (1965), 13 representatives were chosen to compare their results on complementation grouping with ours. They were used as donors and the mutants in our flaA, flaB, $f l a C$, flaD and $f l a J$ were used as recipients. The results are shown in Table 6. Our $A, B, C$ and $D$ fit well with the corresponding group of Joys \& Stocker both in recombination frequency and degree of complementation. Mutants which belong to $f(a E$ by their designation were not present in our mutants, while mutants belonging to our flaJ were not found by them. The grouping of their mutants is comparatively shown in Table 7, column 3. 
The comparable results were obtained in transduction analysis from 11 nonflagellate mutants of Salmonella abortusequi sL 23 to the non-flagellate mutants of S. typhimurium LT2 (Table 7, column 4). Among the non-flagellate mutants of S. abortusequi listed in Table 1, fla-101, -102, -103, -104, -106, -107 and -108 were found to belong to $\mathrm{flaF}$; $f l a-109$ and -110 were to $\mathrm{flaA}$; and $\mathrm{fla}-105$ was $\mathrm{flaC}$. The remaining one, designated by $f a G$, showed unusual behaviour in transduction. Transduction from $f l a G^{-}$to any other $f a^{-}$mutants gave swarms and trails comparable to the transduction from $\mathrm{fla}^{+}$, while when $f l a G^{-}$was used as the recipient none of the transductants were obtained even when $f a^{+}$was used as a donor. The more detailed genetic analysis on flaG mutant is on the way.

It may be worth noting that among the $f l a$ cistrons spontaneous mutants were most abundantly obtained from $f a F$ and next from $f l a A$.

Table 6. Transductions from H 1-linked non-flagellate mutants grouped by Joys \& Stocker (1965) to those grouped by us

Yields of swarms and trails in each combination were graded and listed as Table 2.

\begin{tabular}{|c|c|c|c|c|c|c|c|c|c|c|c|c|c|}
\hline \multirow[b]{3}{*}{ Recipient } & \multicolumn{13}{|c|}{ Donor } \\
\hline & \multicolumn{7}{|c|}{$A$} & \multicolumn{2}{|c|}{$\boldsymbol{B}$} & \multicolumn{2}{|c|}{$C$} & \multirow{2}{*}{$\begin{array}{c}\boldsymbol{D} \\
58\end{array}$} & \multirow{2}{*}{$\begin{array}{c}\boldsymbol{E} \\
60\end{array}$} \\
\hline & 55 & 66 & 52 & 67 & 61 & 57 & 56 & 50 & 59 & 53 & 54 & & \\
\hline$A 37$ & $1(1)$ & $3(3)$ & $3(3)$ & $3(3)$ & $3(3)$ & $2(3)$ & $3(3)$ & $3(3)$ & $3(3)$ & $3(3)$ & $3(3)$ & $\mathbf{3}(\mathbf{3})$ & $3(3)$ \\
\hline 23 & $2(1)$ & $2(0)$ & $1(0)$ & $1(2)$ & $3(3)$ & $2(3)$ & $1(0)$ & $3(3)$ & $3(3)$ & $3(3)$ & $3(3)$ & $3(3)$ & $3(3)$ \\
\hline 34 & $2(2)$ & $2(2)$ & $1(0)$ & $1(0)$ & $2(1)$ & $1(2)$ & $1(0)$ & $3(3)$ & $3(3)$ & $3(3)$ & $3(3)$ & $3(3)$ & $3(3)$ \\
\hline 39 & $2(3)$ & $2(2)$ & $0(0)$ & $1(0)$ & $1(0)$ & $1(1)$ & $0(0)$ & $\mathbf{3}(\mathbf{3})$ & $3(3)$ & $3(3)$ & $\mathbf{3}(\mathbf{3})$ & $3(3)$ & $3(3)$ \\
\hline 41 & $2(2)$ & $0(2)$ & $0(0)$ & $0(0)$ & $0(0)$ & $0(0)$ & $0(0)$ & $\mathbf{3}(\mathbf{3})$ & 3 (3) & 3 (3) & $\mathbf{3}(\mathbf{3})$ & $3(\mathbf{3})$ & $3(3)$ \\
\hline 22 & $3(2)$ & $2(2)$ & $3(3)$ & $1(1)$ & $3(3)$ & $1(1)$ & $0(0)$ & $3(3)$ & $3(3)$ & $3(3)$ & $3(3)$ & $3(3)$ & $3(3)$ \\
\hline B 36 & $3(3)$ & $3(3)$ & $3(3)$ & $3(3)$ & $3(3)$ & $3(3)$ & $3(3)$ & $1(1)$ & $2(1)$ & $3(3)$ & $3(3)$ & $3(3)$ & $3(3)$ \\
\hline$C 21$ & $3(3)$ & $3(3)$ & $3(3)$ & $3(3)$ & $3(\mathbf{3})$ & $3(3)$ & $3(3)$ & $3(3)$ & $3(3)$ & $2(0)$ & $2(2)$ & $3(3)$ & $3(3)$ \\
\hline 38 & $3(3)$ & 3 (3) & $3(3)$ & $3(3)$ & $3(3)$ & $3(3)$ & 3 (3) & $3(3)$ & $3(3)$ & $0(0)$ & $2(2)$ & $3(3)$ & $3(3)$ \\
\hline 31 & $3(3)$ & $3(3)$ & $3(3)$ & $3(3)$ & $3(3)$ & $3(3)$ & $3(3)$ & $3(3)$ & $3(3)$ & $2(0)$ & $2(2)$ & $3(3)$ & $3(3)$ \\
\hline D 42 & 3 (3) & $3(3)$ & $3(3)$ & $3(3)$ & $3(3)$ & $3(3)$ & $3(3)$ & $3(3)$ & $3(3)$ & $3(3)$ & $3(3)$ & $0(0)$ & $3(3)$ \\
\hline $\boldsymbol{J} 28$ & $3(3)$ & $3(3)$ & $3(3)$ & $3(3)$ & $3(3)$ & $3(3)$ & $3(3)$ & $3(3)$ & $3(3)$ & $3(3)$ & $3(3)$ & $3(3)$ & $3(3)$ \\
\hline
\end{tabular}

Table 7. Distribution of non-flagellate mutants among the fla cistrons in Salmonella typhimurium LT2 and $S$. abortusequi $S L 23$

\begin{tabular}{ccccc}
\multicolumn{5}{c}{$\begin{array}{l}\text { fla- mutant of } \\
\text { S. typhimurium } \text { strain LT2 }\end{array}$} \\
cistron & $\begin{array}{c}\text { Listed in } \\
\text { Table } 1\end{array}$ & $\begin{array}{c}\text { Studied by } \\
\text { Joys \& Stocker }\end{array}$ & $\begin{array}{c}\text { S. abortus- } \\
\text { equi }\end{array}$ & Total \\
$\boldsymbol{A}$ & $\mathbf{6}$ & $\mathbf{8}$ & 2 & 16 \\
$B$ & 1 & 2 & 0 & 3 \\
$C$ & 3 & 2 & 1 & 6 \\
$D$ & 1 & 1 & 0 & 2 \\
$E$ & 0 & 1 & 0 & 1 \\
$J$ & 1 & 0 & 0 & 1 \\
$F$ & 9 & 0 & 7 & 16 \\
$G$ & 0 & 0 & 1 & 1 \\
$K$ & 1 & 0 & 0 & 1 \\
Total & 22 & 13 & 11 & 46
\end{tabular}




\section{Antigenicity of the non-flagellate mutants}

The non-flagellate mutants used in the present experiment gave negative reactions in slide-agglutination tests with the anti-H serum prepared against the corresponding original motile strains.

Antisera against each of the twenty-one stable non-flagellate mutants, including 11 strains of Salmonella typhimurium and 10 of $S$. abortusequi which were indicated in Table 1, were prepared by immunizing rabbits with them. The agglutination titres of these antisera to the original flagellate strains were between 6400 and 12,800 . The antisera prepared against non-flagellate mutants originated from LT 2 were absorbed by SL 688; strain SL 688 was derived by two-step transductions of $b$ and enx determinants from $S$. abony CDC 103 to LT 2, thus differing only in $\mathrm{H}$-antigen type from LT2. The antisera to the mutants obtained from sL23 were absorbed by SJ 25; strain sJ25 is an 1,2-type transductional clone in which $H 2^{\text {enx }}$ of sL 23 is replaced by $H 2^{1.2}$ of $\mathrm{LT} 2$. The absorbed antisera were in both cases again titrated with the corresponding original flagellate strains. Among the twenty-one kinds of antisera examined, twenty showed no reaction with the corresponding parental organisms after the absorption at the dilutions ranging from 5 to 12,800 in tube agglutination test. The remaining one, anti-flaG-100 serum absorbed by sJ 25 , had agglutination titre of $\mathbf{5 1 2 0}$ with sL 23, but no agglutination was observed with the cells of $f a G-100$ itself. The entirely parallel results were obtained in the additional two independent series of immunization experiments. The specificity of the anti-enx serum fraction processed from anti-flaG-100 serum was further confirmed by precipitation tests with isolated flagellins of several antigen types: enx-flagellin from sL 23, 1,2 from Salmonella abortusequi sJ 239, and $i$ from monophasic S. typhimurium sw 1166 (Enomoto \& Iino, 1963). Among them only the combination of the antiserum fraction with $e n x$ flagellin gave precipitation band in Ouchterlony test, comparable with that produced between anti-enx serum prepared against SL 23 and enx flagellin. Further, $\mathrm{pH}$ 3-soluble fraction of the disrupted bacteria of $f l a-100$ was found to contain the protein which precipitates specifically with anti-enx serum. Detail of the experiments will be reported elsewhere. Fla-100 organisms, thus produce antigen homologous to that of SL 23 but they cannot form flagella from them, while other non-flagellate mutants do not produce any material cross-reacting with the flagellar antigen. The reversion of $f a G-100$ to $f\left(a^{+}\right.$has never been observed at the order of $10^{8}$ bacteria, and the possibility of the anti-H serum production by motile organisms originated by reversion is excluded.

\section{Test of syntrophism between non-flagellate mutants}

The possibility of syntrophic recovery of flagellation between complementary non-flagellate mutants was examined by mixed culture. Each of the 11 non-flagellate mutants of Salmonella abortusequi were cultivated overnight in broth. One ml. each from two strains was mixed in $10 \mathrm{ml}$. of broth, incubated at $37^{\circ}$ with rotation, and observed at intervals from $2 \mathrm{hr}$ after incubation for 3 days. The mixture was partly brushed on semisolid plates and incubated at $37^{\circ}$ for 3 days. Spreading of the motile organisms was not observed at all. 


\section{DISCUSSION}

By comparing the report by Joys \& Stocker (1965) and our present experiments, sites of non-flagellate mutants were assigned to nine functional units (cistrons) based on complementation. Extensive occurrence of partial complementation in a functional unit leaves the possibility that the minor group, for example flaJ and $f l a K$, each of which is represented by only one mutant, will be placed in another group when non-flagellate mutants are surveyed more extensively in future. Among nine cistrons, six were found to be closely linked to $H 1$; that is, they are clustered in the same chromosomal fragment as $H 1$ in $\mathbf{P} 22$ phage mediated transduction. For the operational convenience, the cluster is termed Fla-1 group. Analysis of recombination obtained by colicine-factor-mediated conjugation showed that $H 1$ and Fla-1 mapped between try and his (Smith \& Stocker, 1962)-though not close enough to be co-transduced with either his or try. The linkage of Fla-1 to a motility cistron and $H 1$ was demonstrated by transduction (Enomoto, unpublished data; Stocker, personal communication). Therefore, it now seems that the Fla-1-H 1 segment and one or more mot loci are close together, and lie between the try and his clusters in the salmonella chromosome. Another non-flagellate mutant fla-29 has been found to be situated between gal and try by colicinoduction (Smith \& Stocker, 1962). This mutant site was found in the present experiment to be in $f l a F$, which is not co-transduced with either $H 1$ or $H 2$. Thus among $H 1$-non-linked fla, flaF, is inferred to be between gal and try. On another H1-non-linked $f l a, f l a K$, chromosomal localization has not been studied yet. The remaining one, flaG, which is represented by one mutant $f a-100$ of Salmonella abortusequi, behaves unusually in transduction experiment; that is, the transduction from the flaGstrain to any other $f a$ strains give flagellated heterogenote and recombinants while the reciprocal transduction fails to give flagellate transductional clones at all. The following possibility may be considered as the nature of flaG- mutant: a multi-site or deletion mutant exceeding its deficient region over the single transduction fragment, a mutant carrying chromosomal abnormality which inhibit pairing or a mutant of cytoplasmic genetic factor. The final conclusion may be reached by the more detailed genetic analysis of the mutant, which is now on the way.

In transduction of flagellation genes between non-flagellate mutants of different serotypes each isolated from nature, Lederberg (1956) noticed that the production of trail in most of the combinations. He posed the question whether the trail production in his experiment meant the presence of many cistrons for flagellation or some special event involved in interserotypic transduction. The present experiment indicated that trail production was observed commonly even in a cistron and that the grouping of the $f a$ mutants by complementation test marking at the trail could be applied even to interserotypic combinations, at least between Salmonella typhimurium, $S$. abortusequi and $\boldsymbol{S}$. paratyphi $\boldsymbol{B}$.

Based on the partial complementation in flaA cistron, Joys \& Stocker divided flaA into five subunits: complementation was observed between the subunits but not in a subunit. In our experiment, complementation in flaA is graded co-linearly to the recombination map sequence. The difference may be partly due to the difference of the scoring method and partly to the difference of the mutants used. Quantitative comparative analysis in future may prove that these differences 
reflect two complementary aspects of a fact. For example, in Table 3 there are two groups of complementation frequencies, all pairs giving either 20-100\% of the number in control crosses or $0-0.5 \%$, and if the former are recorded as positive, the latter as negative, as Joys \& Stocker probably would have done, the data in Table 3 can be represented by the conventional complementation diagram as shown in Fig. 2 (cf. the complementation diagram in Joys \& Stocker, 1965). The latter group of pairs are presumably the ones in which recombination in an abortive transductant is a possible explanation of the very small number of trails observed. Such an event has been demonstrated in transductional heterogenotes of gal genes mediated by phage $\lambda$ in Escherichia coli (Morse, Lederberg \& Lederberg, 1956).

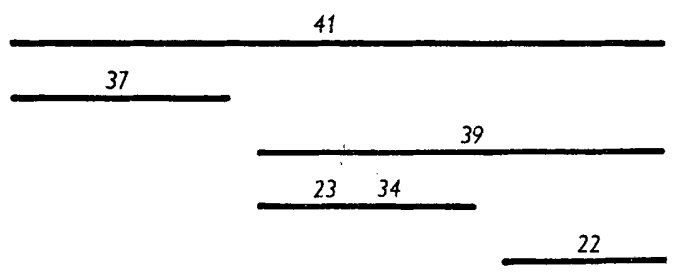

Fig. 2. Complementation diagram of flaA cistron. The complementation frequencies more than $20 \%$ of the control were taken as + , and less than $0.3 \%$ of the control as (cf. Table 3 and Fig. 1).

As regards the functional characteristics, $f l a$ cistrons were divided into two types. The one includes $f a$ other than $f a G$ and the other is represented only by $f a G$. The former group is characterized by the absence of the substance which cross reacts with $\mathrm{H}$-antiserum. A mutation in any one of these cistron from $\mathrm{fla}^{+}$to $\mathrm{fla}^{-}$can cause loss of the ability to synthesize flagella in both flagellar phases. $\mathrm{fla}^{+}$is dominant to $f i a^{-}$in all combinations so far examined. It has been shown that flagellin is a good antigen and has $\mathrm{H}$-antigen specificity of the composing flagella (Ada, Nossal, Pye \& Abbot, 1963). Therefore, the absence of the cross-reacting material may mean that the mutant cannot synthesize at least detectable amounts of flagellin. As the accumulated data have indicated, $H 1$ and $H 2$ are the structural genes of flagellin. fla cistrons of the first group, therefore, are assigned as the regulator genes which control the activity of the flagellin structure genes. As has been discussed in two review articles (Iino \& Lederberg, 1964; Iino, 1964), the most plausible explanation of the function of these $f a$ genes is that they are concerned with the reactions leading to the production of internal inducer. The negative results in the syntrophism test indicate that such inducer, if it is the case, must not be the type of substance which is excreted from a cell and can be incorporated to the other in an active form. The alternative hypothesis still remains as a possibility: that flagellin molecules are synthesized on the specialized active ribosome system (flagellosome) and $f l a$ genes control the production of such specific ribosomes. The second type of $f a, f a G$, must concern to the step of morphogenesis of flagellin into flagella. The presence of specialized flagella-forming apparatus in the flagellate salmonella cells has been predicted (Quadling \& Stocker, 1957; Kerridge, 1960). The flaG mutant may be an excellent material for the studies on the genetic control of such a flagella-forming apparatus. The investigations towards this direction will contribute to the development of genetics of morphogenesis at the level of molecular cytology. 
Contribution from the National Institute of Genetics, Japan, No. 611. This work was partly supported by Public Health Service Research Grant (AI-02872) from the National Institute of Allergy and Infectious Diseases, U.S.A., and by a grant from the Toyo Rayon Foundation for the Promotion of Science and Technology. We thank Professor B. A. D. Stocker and Dr T. M. Joys for exchange of information and the generous provision of bacterial cultures.

\section{REFERENCES}

Ada, G. L., Nossal, G. J., Pye, J. \& Аввot, A. (1963). Behaviour of active bacterial antigens during the induction of the immune response. (1) Properties of flagellar antigens from Salmonella. Nature, Lond. 199, 1257.

Eломото, M. \& Irno, T. (1963a). Colonial dimorphism in non-motile mutants of Salmonella. J. Bact. 86, 473.

Enомото, M. \& Ino, T. (1963b). Chromatography and electrophoresis of flagellin of Salmonella abortus-equi. Rep. natn. Inst. Genet., Misima, 13, 76.

Irvo, T. (1964a). Genetical studies of Salmonella flagella. (in Japanese) Jap. J. Genet. 39, 313.

Iıno, T. (1964b). Genetics of flagellar antigen in Salmonella. Proc. 11th. Congr. Genet. 3, 731.

Inno, T. \& Lederberg, J. (1964). Genetics of Salmonella. In The World Problem of Salmonellosis. Ed. by E. Van Oye, p. 111. The Hague: Dr W. Junk-Publishers.

Joys, T. M. \& Strocker, B. A. D. (1965). Complementation of non-flagellate Salmonella mutants. J. gen. Microbiol. 41, 47.

Kauffmann, F. (1954). Enterobacteriaceae, 2nd ed. Copenhagen: E. Munksgaard.

KerRidge, D. (1960). The effect of inhibitors on the formation of flagella by Salmonella typhimurium. J. gen. Microbiol. 33, 519.

LEDERBERG, J. (1956). Linear inheritance in transductional clones. Genetics, 41, 743.

Lederberg, J. \& IIno, T. (1956). Phase variation in Salmonella. Genetics, 41, 743.

Meyneld, E. W. (1961). A phage, $\phi \chi$, which attacks motile bacteria. J. gen. Microbiol. 25, 253.

Morse, M. L., Lederberg, E. M. \& Lederberg, J. (1956). Transductional heterogenotes in Escherichia coli. Genetics, 41, 758.

Quadling, C. \& Stocker, B. A. D. (1957). The occurrence of rare motile bacteria in some non-motile Salmonella strains. J. gen. Microbiol. 17, 424.

Smith, S. M. \& STocker, B. A. D. (1962). Colicinogeny and recombination. Br. med. Bull. $18,46$.

STOcker, B. A. D. (1956). Abortive transduction of motility in Salmonella; a nonreplicated gene transmitted through many generations to a single descendant. J. gen. Microbiol. 15, 575.

Stocker, B. A. D., Zinder, N. D. \& Lederberg, J. (1953). Transduction of flagellar characters in Salmonella. J. gen. Microbiol. 9, 410. 